\author{
Wing Hung Alex Ng \\ Yu Lung Chan \\ Kit Sum Agnes Au \\ Ka Wai David Yeung \\ Ting Fai Kwan \\ Cho Yee To
}

\section{Morphometry of the corpus callosum in Chinese children: relationship with gender and academic performance}

Received: 21 June 2004

Accepted: 3 August 2004

Published online: 15 October 2004

(C) Springer-Verlag 2004
W. H. A. Ng • Y. L. Chan $(\bowtie)$

Department of Diagnostic Radiology and Organ Imaging, Prince of Wales Hospital, Shatin, Hong Kong, SAR

E-mail: yll90chan@cuhk.edu.hk

Tel.: + 852-26-321002

Fax: + 852-26-360012

Y. L. Chan - K. S. A. Au - K. W. D. Yeung

T. F. Kwan ' C. Y. To

Neurocognitive Science and Language

Education Project, Hong Kong

Present address: K. S. A. Au

Department of Psychology,

James Cook University,

Townsville, Queensland, Australia

\begin{abstract}
Background: The corpus callosum has been widely studied, but no study has demonstrated whether its size and shape have any relationship with language and calculation performance. Objective: To examine the morphometry of the corpus callosum of normal Chinese children and its relationship with gender and academic performance. Materials and methods: One hundred primary school children (63 boys, 37 girls; age 6.5-10 years) were randomly selected and the standardized academic performance for each was ascertained. On the mid-sagittal section of a brain MRI, the length, height and total area of the corpus callosum and its thickness at different sites were measured. These were correlated with sex and academic performance. Results: Apart from the normal average dimension of the different parts of the corpus callo-
\end{abstract}

sum, thickness at the body-splenium junction in the average-to-good performance group was significantly greater than the below-average performance group in Chinese language $(P=0.005)$, English language $(P=0.02)$ and mathematics $(P=0.01)$. The remainder of the callosal thickness showed no significant relationship with academic performance. There was no significant sex difference in the thickness of any part of the corpus callosum.

Conclusions: These findings raise the suggestion that language and mathematics proficiency may be related to the morphometry of the fibre connections in the posterior parietal lobes.

Keywords Brain - Anatomy Corpus callosum ' Intellect . Gender $\cdot$ MRI $\cdot$ Children

\section{Introduction}

The corpus callosum is the main interhemispheric commissure of the brain consisting of approximately 180 million fibres, most of which connect homologous cortical areas. Its functions include unifying sensory fields and organizing bimanual motor output [1], memory [2] and facilitating language and auditory functions [3]. It provides for interhemispheric integration, which is an important function for creativity and intelligence [4]. Language information for writing also seems to flow across the corpus callosum [5]. Global and permanent alexia appears related to lesions involving the splenium [6]. Apraxia is likely related to the flow from the left hemisphere to the right across the posterior corpus callosum [7]. The region of the cerebral cortex that is involved in neural disorders may possibly be deduced from the location of callosal thinning. Callosal abnormalities have been reported in a wide variety of diseases, such as neurofibromatosis [8], spastic diplegia [9], autism, chronic alcoholism, multiple sclerosis [10, 11], Down syndrome [12], cerebrovascular diseases, tumours, chemotherapy and infection. 
The corpus callosum is topographically organized, with projections from the various cortical areas localized to particular regions of this midline structure [13]. The size of the corpus callosum can reflect the abnormalities that originate in the cerebral cortex, and it acts as a sensitive indicator of the cerebral cortical state [14]. However, no study has demonstrated whether the size and shape of the corpus callosum have any relationship with language performance.

The size of the corpus callosum in the postnatal period is largely determined by the degree of myelination of those fibres [13]. Postnatally, the corpus callosum undergoes a tremendous burst of growth during the first 1-4 years, with myelination proceeding in a posterior to anterior sequence $[14,15]$. The study by Yakovlev and Lecours [16] indicated that myelination of the corpus callosum reached adult levels between the ages of 7 and 10 years. However, some studies found increasing callosal area even in the third decade $[17,18]$.

Apart from age, sex differences in callosal morphometry have also been studied. A 'thicker, more bulbous splenium in women than men has been reported [19], but other studies have shown no significant difference in corpus callosal thickness between genders [20, 21]. It has been documented that females tend to outscore males on language-related measures such as reading and vocabulary [22], while males generally display better mathematical ability [23] than females. Nevertheless, even in areas where sex differences are suspected, the differences are subtle and show large variation. For instance, Friedman [24] and Halpern and LaMay [25] showed no male-female differences in general verbal ability and arithmetic skills and argued that none of the evidence for a biological basis for such a difference had been proven. Thus, the relationship between sex differences and language and mathematics performance in terms of neurophysiological data awaits investigation.

The objective of the present study was to assess the morphometry of the corpus callosum in normal Chinese children and evaluate its relationship with sex and academic performance.

\section{Materials and methods}

\section{Subjects}

One hundred primary school children ( 63 boys, age $6.5-$ 10 years, mean 8.08 years; 37 girls, age 6.5-9 years, mean 7.69 years) were recruited to participate in an MRI brain examination program. They were normal Chinese children attending primary I-III classes in formal mainstream primary education in three regional primary schools in Hong Kong. Exclusion criteria for entry into the MRI brain examination included a history of medical, neurological or psychiatric disorders and left-handedness. The study was approved by the Ethics Committee of the Chinese University of Hong Kong. Written informed consent was obtained from the school children and their parents.

All school children in mainstream primary schools in Hong Kong have to participate in an academic performance test (Hong Kong Attainment Tests) in the Chinese language, English language and mathematics standardized by the Hong Kong School Authority. This is a well-established representative assessment of academic performance generally practiced in Hong Kong. The standardized examinations, covering Chinese, English and mathematical skills, are categorized by grades into the 9 years of compulsory and free education in Hong Kong. The tests are administered by individual schools each year (generally in June) to assess the general standards of students at each level. For each subject, pupils' performance from a random sample (based on the date of birth, i.e. roughly 1 of 30 of the total population) of each school is collected by the education authority to compile and monitor the general standard of the year, and for cross-year comparisons and other purposes, e.g. educational research.

One hundred Chinese and English language and 68 mathematics test results were obtained from the school authorities. Mathematics test results were not available in the youngest group of 32 children in primary I, as the standardized mathematics test was not, as a rule, applied to primary I students. The performances were allotted to an average-to-good performance group and a belowaverage performance group using the median result as the discriminator.

\section{MRI technique}

The MRI examination was performed using a 1.5-T imager (Gyroscan ACS NT, Philips Medical Systems, Best, The Netherlands) using a standard head coil. From the survey scans, T2-weighted (T2-W) mid-sagittal sections through the anterior and posterior commissures and axial sections through the third ventricle were obtained for measurements. The $\mathrm{T} 2-\mathrm{W}$ images were acquired using the following parameters: TR/TE 3,000/ $120 \mathrm{~ms}$, slice thickness $3 \mathrm{~mm}$, matrix $128 \times 256$, number of excitations 3 , field of view $230 \mathrm{~mm}$.

\section{Image analysis}

The total area of the corpus callosum was measured by a manual tracing method using the mid-sagittal image (Fig. 1). The most inferior points of the rostrum and splenium were connected by a tangent (A). This line served as the reference line for the subsequent steps [26]. 


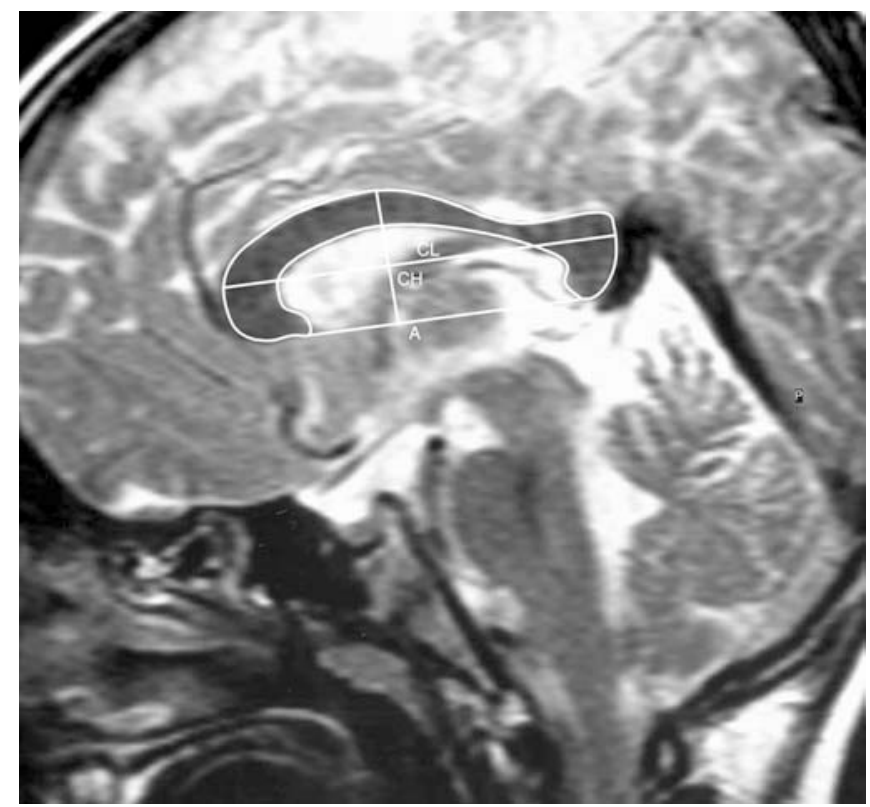

Fig. 1 The mid-sagittal section is defined as the image through the anterior and posterior commissures. The total area of the corpus callosum was measured by manual tracing. The most inferior points of rostrum and splenium were connected by a tangent (A). This line served as a reference line for the subsequent steps. $\mathrm{CH}$ was the maximum length of the corpus callosum perpendicular to line A. CL was the maximum length of the corpus callosum parallel to line A

The callosal height $(\mathrm{CH})$ was the maximum length of the corpus callosum perpendicular to line A. The callosal length $(\mathrm{CL})$ was the maximum length of the corpus callosum parallel to line A. Because the corpus callosum shows high topographical variability, Weis et al. [26] divided the corpus callosum into equidistant fifths by vertical lines. The first fifth corresponds to the genu; the second, third and fourth fifths correspond to the anterior, mid and posterior parts of the body; the final fifth corresponds to the splenium (Fig. 2). The thickness of the genu (CLA) and splenium (CLF) were defined as the maximum width of the genu and splenium parallel to line A. The thickness of the body perpendicular to line A was measured at its anterior junction with the genu (CLB), at the junction of the anterior third and posterior two-thirds (CLC), at the junction of the anterior twothirds and the posterior third (CLD), and at its junction with the splenium (CLE).

Total intracranial volume was estimated by modelling the head as an oblate spheroid [27]. This analysis was based on the distance between inner skull margins on three brain slices to represent $x, y$ and $z$ axes. The $x$ and $y$-axis lengths were defined as the left and right extreme and anterior to posterior extreme respectively from an axial slice at the level of the third ventricle. The $z$-axis length was defined as the superior margin of the brain to the line projected from the anterior to posterior

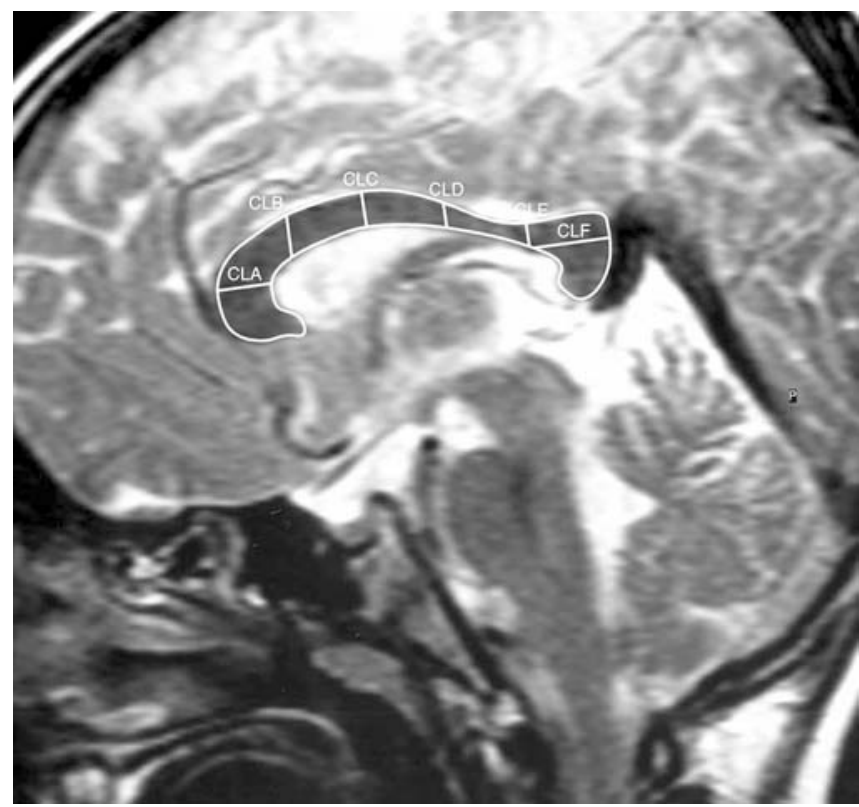

Fig. 2 The corpus callosum was divided into five parts by equidistant vertical lines. The thickness of the genu (CLA) and splenium (CLF) were defined as the maximum extension of the genu and splenium parallel to line A. The thickness at the junction of the divided parts was measured: CLB junction of genu and body, CLC junction of anterior third and posterior two-thirds of the body, CLD junction of anterior third and posterior two-thirds of the body, CLE junction of body and splenium

clivus in the mid sagittal section. The resulting voxel count was then transformed into a volume $(\mathrm{ml})$ using the formula: volume $=4 / 3 \times \pi \times X / 2 \times Y / 2 \times Z / 2$. The adjusted parameters of the corpus callosum were defined as true parameters of the corpus callosum divided by the intracranial volume.

\section{Statistical analysis}

Age differences in the parameters of the corpus callosum, with and without intracranial volume adjustment, were assessed by Pearson correlation. Sex differences in the parameters of the corpus callosum, with and without intracranial volume adjustment, were assessed by Student's $t$-test. Sex differences in the academic results were also evaluated by Student's $t$-test. To evaluate the relationship of the morphometry of the corpus callosum with academic performance, Student's $t$-test was employed. For all statistical analyses, a two-tailed $P$ value of $<0.05$ was regarded as significant. The parameters of the corpus callosum were compared with and without intracranial volume adjustment.

Interobserver variation was assessed by another researcher who measured 40 randomly selected children. The correlation coefficient was used for assessment. 
Table 1 Linear dimension of different parts of corpus callosum

\begin{tabular}{llll}
\hline & $\begin{array}{l}\text { Boys, } \\
n=63(\mathrm{~mm})\end{array}$ & $\begin{array}{l}\text { Girls, } \\
n=37(\mathrm{~mm})\end{array}$ & $\begin{array}{l}\text { All children, } \\
n=100(\mathrm{~mm})\end{array}$ \\
\hline CLA & $9.41( \pm 1.56)$ & $9.25( \pm 1.31)$ & $9.35( \pm 1.47)$ \\
CLB & $6.58( \pm 1.27)$ & $6.19( \pm 1.42)$ & $6.44( \pm 1.33)$ \\
CLC & $5.59( \pm 0.98)$ & $5.33( \pm 0.80)$ & $5.49( \pm 0.92)$ \\
CLD & $4.60( \pm 0.88)$ & $4.40( \pm 1.07)$ & $4.53( \pm 0.96)$ \\
CLE & $6.40( \pm 1.39)$ & $5.89( \pm 1.83)$ & $6.21( \pm 1.58)$ \\
CLF & $8.98( \pm 1.47)$ & $8.70( \pm 1.49)$ & $8.88( \pm 1.47)$ \\
CL & $60.92( \pm 5.16)$ & $60.50( \pm 6.56)$ & $60.77( \pm 5.69)$ \\
CH & $22.11( \pm 2.76)$ & $21.10( \pm 3.14)$ & $21.74( \pm 2.93)$
\end{tabular}

The thickness of the genu (CLA), body at its anterior junction with the genu (CLB), at the junction of the anterior third and posterior two-thirds (CLC), of the anterior two-thirds and the posterior third (CLD), the posterior junction with the splenium (CLE), and splenium (CLF)

Dimension given as mean $\pm \mathrm{SD}$

$C L$ callosal length, $\mathrm{CH}$ callosal height

\section{Results}

The average total callosal area in the mid-sagittal plane measured $538.1 \pm 17.3 \mathrm{~mm}^{2}$. The average $\mathrm{CL}$ was $60.8 \pm 1.12 \mathrm{~mm}$ and the $\mathrm{CH}$ was $21.7 \pm 0.57 \mathrm{~mm}$. Table 1 illustrates the thicknesses of the different segments of the corpus callosum. No statistically significant difference was shown between the two independent observers for all measurements in the 40 randomly selected subjects. The interobserver correlation coefficients for $\mathrm{CH}, \mathrm{CL}$ and thickness, callosal area and estimated intracranial volume for the 40 randomly selected subjects were $0.88,0.99,0.92$ and 0.87 , respectively. The results indicate strong interobserver agreement with regard to the morphometric measurements.

Age differences in different parameters of the corpus callosum

There was no significant correlation between age and $\mathrm{CH}(r=0.039, P=0.7)$ and length of the corpus callosum $(r=0.039, P=0.700)$ in the age range studied. The total callosal area also showed no significant age differences $(r=0.134, \quad P=0.18)$. Considering callosal thickness, there was a weak but significant correlation between CLC and age $(r=0.201, P=0.045)$. The thickness of the remainder of the corpus callosum showed no significant correlation with age $(r=0.018-0.180$, $P>0.05)$.

Sex differences in different parameters of corpus callosum and academic performance

There was no significant sex difference in the height $(\mathrm{CH})$ either with $(P=0.231)$ or without $(P=0.096)$ intracranial volume adjustment. There was also no significant sex difference in the length $(C L)$ with $(P=0.352)$ or without $(P=0.731)$ intracranial volume adjustment. Total callosal area (CC) also showed no significant sex difference $(P=0.235)$, a relationship maintained after adjustment for intracranial volume $(P=0.495)$. There was no significant sex difference for the thickness of different portions of the corpus callosum $(P>0.05)$, with or without intracranial volume adjustment.

There were no significant sex differences in academic performance in Chinese $(P=0.09)$, English $(P=0.19)$ or mathematics $(P=0.11)$.

Academic performance difference in different parameters of corpus callosum

Table 2 shows the average thickness of different segments of the corpus callosum in the average-to-good and below-average performance groups in Chinese, English and mathematics. There were significant differences for CLE between the average-to-good and below-average performers in Chinese $(P=0.005)$, English $(P=0.019)$ and mathematics $(P=0.011)$. The other parameters, including thickness of the other body segments, total area, height and length of corpus callosum, showed no significant difference $(P>0.05)$ between the two groups.

\section{Discussion}

The corpus callosum provides for interhemispheric integration, which is an important function for creativity

Table 2 Thickness of different parts of corpus callosum in relation to academic performance in English, Chinese and mathematics

\begin{tabular}{|c|c|c|c|c|c|c|c|c|c|}
\hline $\begin{array}{l}\text { Thickness } \\
(\mathrm{mm})\end{array}$ & $\begin{array}{l}\text { Good } \\
\text { English }\end{array}$ & $\begin{array}{l}\text { Poor } \\
\text { English }\end{array}$ & $\begin{array}{l}t \text {-test } \\
(P \text {-value })\end{array}$ & Good Chinese & Poor Chinese & $\begin{array}{l}t \text {-test } \\
(P \text {-value })\end{array}$ & $\begin{array}{l}\text { Good } \\
\text { Mathematics }\end{array}$ & $\begin{array}{l}\text { Poor } \\
\text { Mathematics }\end{array}$ & $\begin{array}{l}t \text {-test } \\
(P \text {-value })\end{array}$ \\
\hline CLE & $6.58 \pm 1.55$ & $5.85 \pm 1.53$ & 0.019 & $6.64 \pm 1.55$ & $5.77 \pm 1.49$ & 0.005 & $6.52 \pm 1.56$ & $5.52 \pm 1.59$ & 0.011 \\
\hline CLA & $9.19 \pm 1.46$ & $9.51 \pm 1.48$ & 0.281 & $9.58 \pm 1.52$ & $9.11 \pm 1.39$ & 0.104 & $9.87 \pm 1.60$ & $9.21 \pm 1.35$ & 0.700 \\
\hline CLB & $6.33 \pm 1.31$ & $6.54 \pm 1.35$ & 0.434 & $6.65 \pm 1.47$ & $6.21 \pm 1.14$ & 0.096 & $6.65 \pm 1.56$ & $6.31 \pm 1.31$ & 0.344 \\
\hline CLC & $5.37 \pm 0.89$ & $5.62 \pm 0.95$ & 0.182 & $5.63 \pm 0.88$ & $5.35 \pm 0.95$ & 0.137 & $5.47 \pm 1.01$ & $5.56 \pm 1.00$ & 0.709 \\
\hline CLD & $4.53 \pm 1.07$ & $4.52 \pm 0.84$ & 0.967 & $4.55 \pm 1.09$ & $4.50 \pm 0.80$ & 0.760 & $4.55 \pm 0.86$ & $4.27 \pm 0.81$ & 0.168 \\
\hline CLF & $8.89 \pm 1.51$ & $8.97 \pm 1.44$ & 0.535 & $8.98 \pm 1.34$ & $8.78 \pm 1.60$ & 0.489 & $8.77 \pm 1.49$ & $8.77 \pm 1.53$ & 1.00 \\
\hline
\end{tabular}

Dimension given as mean $\pm \mathrm{SD}$ 
and intelligence [4]. The functional topography of the fibres in the human corpus callosum is still not fully understood. The temporal, parietal and occipital lobe fibres are believed to cross in the splenium and the caudal part of the corpus callosum [28-30]. Language information for writing seems to flow across the corpus callosum [31]. Of similar functional interest is the posterior body of the corpus callosum, which carries fibres connecting the posterior parietal and temporal regions of the two hemispheres involved in language function $[32,33]$. Several clinical studies have also alluded to this hypothesis. Global and permanent alexia and apraxia, as well as other disorders of language, have been shown to relate to the interference of flow from the left hemisphere to the right across the posterior corpus callosum $[6,7]$. Egaas et al. [34] examined 51 autistic patients (age 342 years) and found that the overall size reduction in the corpus callosum was concentrated in the posterior subregions where parietal lobe fibres are known to project [13]. As the symptoms of autism include limited verbal and non-verbal communication, an association of language performance and size of the posterior corpus callosum is suspected. Our findings of a significant difference in the thickness of the posterior part of the corpus callosum with respect to language performance in the standardized tests in Chinese and English are consistent with the hypothesis that language function may be related to the structural make-up of the corpus callosum.

The thicker posterior body of the corpus callosum is also associated with better performance in mathematics. Mathematical intuition may depend on the interplay of both word association and visual-spatial processing, the network of which both involve the parietal lobes [35]. Processing Arabic digits has been shown by PET to produce activation of the occipitoparietal areas bilaterally [36]. fMRI studies reveal activation unique to calculation in both inferior parietal lobules [37]. Bilateral intraparietal areas show greater activation on fMRI during approximation, whereas bilateral angular regions are more active during exact calculation [38]. The bilateral nature of the neural substrates in the parietal and parieto-occipital regions involved in mathematical skills underlies the role of the interconnecting fibres in the posterior part of the corpus callosum. Our findings also suggested that the area of the corpus callosum involved in calculation may be the posterior part of the corpus callosum, i.e. where the posterior parietal, occipital and temporal lobes on both sides are interconnected. Although the corpus callosum has been suggested to have an important function for creativity and intelligence [4], the current study is, to our knowledge, the first to evaluate the relationship of calculation and the morphometry of corpus callosum.

Quantitative electron microscopy studies in the rhesus monkey indicate that new callosal axons do not develop postnatally [39]. This reasonably suggests that myelination is the most likely reason for age-related increase in the size of the corpus callosum in humans. The association of academic performance with the thickness of the posterior body of the corpus callosum may be explained by the functional changes that accompany myelination of axons traversing the corpus callosum. Myelination of axons determines the speed of pulse transmission along the nerve fibres. The current findings of a significant relationship between the posterior corpus callosal thickness and language and mathematics performance may be accounted for by the thicker and better myelinated posterior part of the corpus callosum, allowing for higher speed of nerve transmission and a more efficient neural network concerned with the task. The myelination of fibre tracts around the peritrigonal region involving the association areas of the posterior and inferior parietal and posterior temporal cortex is known to be delayed in the first decade, with a possible delay up to the second decade, as compared to the rest of the cerebral hemispheric white-matter tracts [40]. It is likely that the interconnecting fibres in the posterior corpus callosum would show a similar pattern of delay in myelination, subject to individual variation.

In the present study, there was no significant relationship between the total callosal area and age from 6.5 to 10 years old. Only the subdivision of thickness of the corpus callosum at the mid-portion (CLC) showed significant increase with age. This suggests that age is not a confounding factor and the thicker the posterior part of the corpus callosum, the better the academic performance. Thompson et al. [41] have found that in subjects aged $6-15$ years, the highest growth rates were consistently attained in temporoparietal systems which are functionally specialized for language and for understanding spatial relations [41]. The range of age of the school children in our study may be too narrow for the effect of this increase in size to be detected, except at the mid-portion where the most rapid increase in size may have occurred. A larger sample may be needed to evaluate further the increase in size of the corpus callosum in the narrow age range targeted in the present study. However, our results do imply that from 6 to 10 years old, the mid-portion in the corpus callosum contributes most of the development of that structure.

The current study demonstrated no significant sex difference in the morphometry of the corpus callosum in children between 6 and 10 years old, which is consistent with the childhood findings of Giedd et al. [4]. Allen et al. [42] also found no sex difference in the corpus callosal area or its subdivisions in children from 2 to 16 years old. However, they suggested that the shape of the splenium was consistently wider or more bulbous in girls than boys. Clarke et al. [43] evaluated 28 brains derived from autopsy, ranging from 20 weeks' gestation to 14 years old and found that boys had significantly 
larger corpus callosal area than girls. The discrepancy with our results may arise from the differential growth of the corpus callosum in the different periods of childhood between the two sexes. The lack of significant sex differences in corpus callosum morphometry in the current study does not provide morphometric evidence (at least in the corpus callosum) for neurophysiological differences between males and females proposed for their differential performance in language and mathematical skills.

In conclusion, there is a significant relationship between the thickness of posterior body of the corpus callosum and academic performance in Chinese language, English language and mathematics. The finding suggests that language and mathematics proficiency is related to the morphometry of the fibres interconnecting both posterior parietal lobes and both temporal lobes. Whether the better performance associated with a thicker posterior body of the corpus callosum is due to differences in myelination of these crossing fibres has to be addressed by further study. The present study does not lend support for a morphometrical callosal difference between males and females to account for their differential performance in language and mathematics.

Acknowledgements This work was supported by grant number 1999/0308 from the Quality Education Fund, Hong Kong, SAR.

\section{References}

1. Zaidel D, Sperry RW (1977) Some longterm effects of cerebral commissurotomy in man. Neuropsychology 15:193204

2. Zaidel D, Sperry RW (1974) Memory impairment after commissurotomy in man. Brain 97:263-272

3. Schaltenbrand G (1975) The effects on speech and language of stereotactical stimulation in thalamus and corpus callosum. Brain Lang 2:70-77

4. Giedd JN, Blumenthal J, Jeffries NO, et al (1999) Development of the human corpus callosum during childhood and adolescence: a longitudinal MRI study. Prog Neuropsychopharmacol Biol Psychiatry 23:571-588

5. Geschwind N (1965) Disconnection syndromes in animals and man. Brain 88:585-644

6. Howard D, Patterson K, Wise R, et al (1996) A chronic microelectrode investigation of the tonotopic organization of the human auditory cortex. Brain Res 734:260-264

7. Kawamura M, Hirayama K, Yamamoto H (1989) Different interhemispheric transfer of kanji and kana writing evidenced by a case with left unilateral agraphia without apraxia. Brain 112:1011-1018

8. Dubovsky EC, Booth TN, Vezina G, et al (2001) MR imaging of the corpus callosum in pediatric patients with neurofibromatosis type 1 . AJNR 22:190-195
9. Iai M, Tanabe Y, Goto M, et al (1994) A comparative magnetic resonance imaging study of the corpus callosum in neurologically normal children and children with spastic diplegia. Acta Paediatr 83:1086-1090

10. Pozzilli C, Bastinanello S, Padovanni A, et al (1991) Anterior corpus callosum atrophy and verbal fluency in multiple sclerosis. Cortex 27:441-445

11. Gean-Marton AD, Vezina LG, Marton KI, et al (1991) Abnormal corpus callosum: a sensitive and specific indicator of multiple sclerosis. Radiology 180:215-221

12. Wang PP, Doherty S, Hesselink JR, et al (1992) Callosal morphology concurs with neurobehavioral and neuropathological findings in two neurodevelopmental disorders. Arch Neurol 49:407-411

13. DeLacoste MC, Kirkpatrick JB, Ross ED (1985) Topography of the human corpus callosum. J Neuropathol Exp Neurol 44:578-591

14. Hayakawa K, Konishi Y, Matsuda T, et al (1989) Development and aging of brain midline structures: assessment with MR imaging. Radiology 172:171177

15. Georgy BA, Hesselink JR, Jernigan TL (1993) MR imaging of the corpus callosum. AJR 160:949-955

16. Yakovlev PI, Lecours AR (1967) The myelogenetic cycles of regional maturation of the brain. In: Minkowski A (ed) Regional development of the brain in early life. Blackwell Scientific, Oxford pp 3-70

17. Pujol J, Vendrell P, Junque C (1993) When does human brain development end? Evidence of corpus callosum growth up to adulthood. Ann Neurol 34:71-75
18. Rauch RA, Jinkins JR (1994) Analysis of cross-sectional area measurements of the corpus callosum adjusted for brain size in male and female subjects from childhood to adulthood. Behav Brain Res 64:65-78

19. De-Lacoste-Utamsing C, Holloway RL (1982) Sexual dimorphism in the human corpus callosum. Science 216:1431-1432

20. Pozzilli C, Bastianello S, Bozzao A, et al (1994) No differences in corpus callosum size by sex and aging. A quantitative study using magnetic resonance imaging. J Neuroimaging 4:218-221

21. Steinmetz H, Jancke L, Kleinschmidt A, et al (1992) Sex but no hand difference in the isthmus of the corpus callosum. Neurology 42:749-752

22. Halpern DF (2000) Sex differences in cognitive abilities. Erlbaum, Mahwah

23. Stumpf H, Stanley JC (1996) Genderrelated differences on the College Boards' Advanced Placement and Achievement Tests, 1982-1992. J Edu Psychol 88:353-364

24. Friedman L (1995) The space factor in mathematics: gender differences. Rev Educ Res 65:22-50

25. Halpern DF, LaMay ML (2000) The smarter sex: a critical review of sex differences in intelligence. Edu Psychol Rev 12:229-246

26. Weis S, Kimbacher M, Wenger E, et al (1993) Morphometric analysis of the corpus callosum using MR: correlation of measurements with aging in healthy individuals. AJNR 14:637-645 
27. Sullivan EV, Rosenbloom MJ, Desmond JE (2001) Sex differences in corpus callosum size: relationship to age and intracranial size. Neurobiol Aging 22:603-611

28. Carpenter MB (1990) Core text of neuroanatomy, 4th edn. Williams and Wilkins, Baltimore, p 37

29. Barkovich AJ, Kjos BO (1988) Normal postnatal development of the corpus callosum as demonstrated by MR imaging. AJNR 9:487-491

30. Barkovich AJ, Norman D (1988) Anomalies of the corpus callosum: correlation with further anomalies of the brain. AJR 51:171-177

31. Geschwind N, Levitsky W (1968) Human brain: left-right asymmetry in temporal speech region. Science 161:186-187

32. Seltzer B, Pandya DN (1983) The distribution of posterior parietal fibers in the corpus callosum of the rhesus monkey. Exp Brain Res 49:147-150
33. Cipolloni PB, Pandya DN (1985) Topography and trajectories of commissural fibers of the superior temporal region in the rhesus monkey. Exp Brain Res 57:381-389

34. Egaas B, Courchesne E, Saitoh O (1995) Reduced size of corpus callosum in autism. Arch Neurol 52:794-801

35. Dehaene S, Spelke E, Pinel P, et al (1999) Sources of mathematical thinking: behavioral and brain-imaging evidence. Science 284:970-974

36. Pesenti M, Thioux M, Seron X, et al (2000) Neuroanatomical substrates of Arabic number processing, numerical comparison, and simple addition: a PET study. J Cogn Neurosci 12:461-79

37. Simon O, Mangin JF, Cohen L, et al (2002) Topographical layout of hand, eye, calculation, and language-related areas in the human parietal lobe. Neuron $33: 475-487$

38. Stanescu-Cosson R, Pinel P, van De Moortele PF, et al (2000) Understanding dissociations in dyscalculia: a brain imaging study of the impact of number size on the cerebral networks for exact and approximate calculation. Brain 123:2240-2255
39. LaMantia AS, Rakic P (1990) Cytological and quantitative characteristics of four cerebral commissures in the rhesus monkey. J Comp Neurol 291:520-537

40. Barkovich AJ (1995) Pediatric neuroimaging. Raven Press, New York, pp 952

41. Thompson PM, Giedd JN, Woods RP, et al (2000) Growth patterns in the developing brain detected by using continuum mechanical tensor maps. Nature 404:190-193

42. Allen LS, Richey MF, Chai YM, et al (1991) Sex differences in the corpus callosum of the living human being. J Neurosci 11:933-942

43. Clarke S, Kraftsik R, Van der Loos H, et al (1989) Forms and measures of adult and developing human corpus callosum: is there sexual dimorphism? J Comp Neurol 280:213-230 